\title{
Proceedings of the 132nd Semon Club, Otolaryngology Department, Guy's and St Thomas' NHS Foundation Trust, London, UK, 17 November 2006
}

\author{
Chairman: Mr Ricard Simo, Consultant Otorhinolaryngologist and Head \& Neck Surgeon, Guy's and St Thomas' NHS Foundation Trust, \\ London \\ Pathologists: Professor Leslie Michaels and Dr Ann Sandison, University College London \\ Radiologist: Dr Steve Connor, Consultant Radiologist, Guy's and St Thomas' NHS Foundation Trust, London \\ A prize was awarded for the best presentation of the meeting
}

\section{Otology and skull base session}

Chairman: Mr Tony Aymat

\section{Chronic pain with a bone-anchored hearing aid: histological findings}

D H Gadhvi, A Dezso, A F Fitzgerald-O'Connor

From St Thomas' Hospital, London, UK

Case report

A 60-year-old woman with Treacher-Collins syndrome had previously undergone bilateral exploratory tympanotomies and a left stapedectomy. She had a chronically discharging left ear and meatal stenosis on the right. A bone-anchored hearing aid (BAHA) fixture had been implanted in March 2005. Post-operatively, she had developed wound infections that were successfully treated with antibiotics. She was a good user of the implant and the surrounding skin became stable. Unfortunately, she developed chronic pain that was resistant to treatment. The fixture was removed in May 2006 with a core of bone surrounding the implant.

\section{Discussion}

Professor Michaels identified a trapped hair follicle under the fixture. He also pointed out a cluster of plasma cells and a foreign body reaction to a single hair shaft. At the surface of the titanium fixture, there was evidence of bone loss and failure of bone growth, which indicated that the implant was not fully osseointegrated. Titanium implants have been used in the craniofacial region for over two decades. A minority of patients with BAHAs suffer with chronic pain. Further research is necessary to investigate this phenomenon.

\section{An unusual facial paralysis}

S Patil, S Davis, S Habashi

From the Chase Farm Hospital, Enfield, UK

\section{Case report}

A 32-year-old Turkish man presented with a five-day history of left facial palsy. Associated otorrhoea and otalgia of 15 days' duration was noted. A past history of decreased hearing and perforation of one year's duration was present. The patient was being treated for familial Mediterranean fever, AA amyloid nephropathy and endstage renal failure.

On examination, a grade VI facial paresis was noted. Otoscopy revealed polypoid tissue in the external auditory canal. An urgent canal wall down tympanomastoidectomy with facial nerve decompression was performed. It was noted that the middle ear was filled with gelatinous, polypoid material. The facial nerve was dehiscent in its horizontal part and was oedematous and thickened. Postoperatively, the patient recovered full facial nerve function in a month.

\section{Histology}

Histopathological analysis revealed polypoid, inflammatory granulation tissue containing plasma cells and neutrophils, in keeping with otitis media and a benign aural polyp.

\section{Discussion}

Familial Mediterranean fever has a highly variable presentation rate (1:1000 to 1:70 000). Development of facial palsy is a very rare complication of this disease. This patient presented dilemmas in diagnosis and management, as he had amyloidosis, chronic renal failure, diabetes mellitus and chronic serous otitis media. The question was posed of whether amyloid changes in the facial nerve predisposed to acute facial nerve palsy.

\section{Importance of imaging the central auditory pathways in cases of unilateral sensorineural hearing loss}

J M Fishman, M Shoeb, S Abramovich*

From Northwick Park Hospital, Harrow, and the *Central Middlesex Hospital, London, UK

\section{Case report}

Adults with asymmetrical or unilateral sensorineural hearing loss (SNHL) often undergo imaging of the internal auditory meatus (IAM) to evaluate for cochlear or retrocochlear abnormalities. Much emphasis is placed on imaging the IAM, but often little attention is given to imaging the higher auditory pathways.

We report three cases of adults presenting with a predominantly unilateral SNHL. Hearing loss was the sole initial presenting complaint in all cases. Imaging revealed a large meningioma in one patient, a demyelinating process in the second and evidence of widespread vasculitis in the third.

In all cases, hearing loss was presumed to be associated with the observed pathology, although the exact mechanisms responsible for the SNHL remained elusive. In all cases, the SNHL appeared to have resulted from either a direct effect on the auditory nerves or central auditory pathways, rather than a direct effect on the cochlea. 


\section{Conclusion}

In addition to imaging the IAM, the entire acoustic pathway from the cochlea to the superior temporal (Heschl's) gyrus should be scrutinised for lesions in patients with unilateral SNHL. The aim should be not only to exclude specific cochlear or retrocochlear aetiologies, but also to exclude central abnormalities, by using appropriate imaging techniques.

\section{'Doctor, I can't hear anything. By the way, I have HIV' \\ C J Skilbeck, A F Fitzgerald-O'Connor \\ From St Thomas' Hospital, London, UK}

\section{Case report}

We report the case of a 44-year-old man who presented with bilateral hearing loss which progressed over several days. He had no otalgia or otorrhoea, but on direct questioning did report unilateral tinnitus. He had no vertigo.

The patient had previously been diagnosed as human immunodeficiency virus (HIV) positive but had never received anti-retroviral medication. At the time of the current presentation, he was not taking any medication, although he had recently taken a single dose of tinidazole for giardiasis.

On examination, both external auditory canals and tympanic membranes appeared normal. The cranial nerve examination was normal except for VIII. There was no nystagmus. The Romberg and Unterberger tests were negative.

Pure tone audiometry showed bilateral profound sensorineural hearing loss. Tympanometry was normal in both ears.

Magnetic resonance imaging of the patient's skull base showed prominent enhancement of both cochleas.

\section{Discussion}

After discussion with the local HIV team, the patient was transferred for further management. He was started on empirical treatment with acyclovir and prednisolone, as well as combination highly active anti-retroviral medication. Investigations included full blood count, electrolytes, specific serology, viral load, cluster of differentiation 4 glycoprotein (CD4) count and lumbar puncture.

The only abnormal result was positive serology for syphilis. The patient had been tested for syphilis four months previously, with a negative result. He was therefore diagnosed with otosyphilis and commenced on intravenous penicillin.

The meeting discussed the clinical, pathological and management issues of syphilis-related hearing loss.

Finally, the long term implications for this patient were discussed.

\section{Mixed hearing loss and facial paralysis following barotrauma}

A Hassaan, J Manickavasagam, A K Battacharyya

From Whipps Cross University Hospital, London, UK

\section{Case report}

A 52-year-old man presented stating that a firecracker had exploded near his right ear. He reported immediate dizziness, tinnitus, reduction in hearing and complete weakness of the right side of the face.

Examination yielded a right ear laceration and blood clots within the canal. The tympanum was not visualised. There was grade one nystagmus and a right grade five (HouseBrackmann) facial palsy. The left ear appeared normal.
At this point, Dr Connor pointed out evidence of ossicular discontinuity on the computed tomography scans displayed.

Intra-operative findings were a posterior tympanic perforation, a dislocated stapes impinging on a dehiscent facial nerve surrounded by haematoma, and a hypermobile incus. The incus, stapes and haematoma were removed, the facial nerve sheath opened, a temporalis fascia graft to the oval window performed and prosthetic ossicular reconstruction undertaken.

Electromyography at post-operative week seven indicated complete lesion of the right facial nerve, with no evidence of functioning motor axons.

\section{Discussion}

Mr Roberts enquired as to the incidence of this condition. Our literature search had identified no other cases. A colleague raised the issue of decompressing the facial nerve canal proximally where it is narrowest; the advantages and disadvantages of this procedure were discussed.

\section{Head and neck session}

Chairman: Mr Jean-Pierre Jeannon

\section{Anaplastic lymphoma presenting as tracheoesophageal fistula}

A Joshi, P Fields, R Simo

From Guy's Hospital, London, UK

\section{Case report}

We report the case of a 56-year-old man referred to the head and neck unit of our tertiary referral hospital with a three-week history of sore throat, odynophagia, dysphagia, dysphonia and loss of weight. He was initially reported to have a pharyngeal pouch which had been diagnosed at oesophagogastroduodenoscopy two weeks prior to the referral.

On endoscopy, we found a tracheoesophageal fistula through a mass in the anterior oesophageal wall. A biopsy of the ulcerative lesion was taken. The patient subsequently underwent a computed tomography scan of his neck and chest. Histological analysis of the biopsy revealed anaplastic lymphoma. The patient was referred to the oncology unit and treated with chemotherapy.

Kinase-1 positive (ALK-1) anaplastic lymphoma is a very rare but distinct pathological entity, within the group of anaplastic large cell lymphomas. This condition affects, in decreasing order of frequency, the bone marrow, skin, lungs and soft tissue. Involvement of the gastro-intestinal tract is very rare; even if present, it is only seen as an associated feature, together with other, more predominantly involved systems. Since there has been no previously reported case with involvement of the oesophagus as the presenting feature, we took this opportunity to present this very rare condition.

Massive cervicothoracic haematoma due to extracapsular rupture of asymptomatic parathyroid adenoma

S S Vasani, V Prasad, A Trinidade

From the Royal Free Hospital, London, UK

\section{Case report}

A 56-year-old woman presented with severe spontaneous cervical ecchymosis with difficulty in breathing and sudden onset dysphagia, following an upper respiratory tract infection and 24 hours of cephalosporin use. Flexible nasendoscopy revealed bilateral false vocal fold haematomas. The patient was resuscitated and treated 
symptomatically. Clotting studies revealed no abnormality but biochemical investigations showed mildly elevated serum calcium and parathormone levels. Computed tomography scanning showed significant retro-tracheal haemorrhage. A methoxyisobutyl isonitrile (MIBI) scan was performed which confirmed the presence of a right parathyroid adenoma. At the time of writing, the patient was awaiting excision of a parathyroid adenoma.

\section{Discussion}

The difficulty in establishing this diagnosis was discussed. Extra-capsular rupture of an enlarged, hyperplastic parathyroid gland or adenoma is a rare cause of unexplained cervical or mediastinal haemorrhage, which in some cases can be fatal. Early diagnosis and management of this condition can be difficult, as there are usually no obvious abnormalities detected on biochemical, haematological and radiological investigation. The need for quick and effective primary management, and the low but definite risk of mortality due to airway obstruction, necessitate a high degree of clinical suspicion in these cases.

\section{Toxoplasma lymphadenitis of the parotid gland}

A Najuko, J R Tysome, C Barbaccia

From the Medway Maritime Hospital, Gillingham, UK

\section{Case report}

A 40-year-old man presented with a two-month history of a painless, $1.5 \mathrm{~cm}$, left pre-auricular mass and a $1 \mathrm{~cm}$ upper neck mass. His facial nerve was intact. Ultrasound imaging confirmed intra-parotid lymph nodes. Fine needle aspiration cytology revealed a mixed lymphocytic picture. Laboratory test results were within normal limits. Since both masses had persisted for several weeks, a left superficial parotidectomy was performed. Histopathological findings were characteristic of toxoplasma lymphadenitis. Subsequent serological tests confirmed this diagnosis. There was no history of exposure to cats.

\section{Discussion}

Despite a prevalence of 33 per cent in adults in the UK, toxoplasmosis has rarely been reported in the parotid gland. Most cases have been diagnosed from the histology of the resected gland.

Professor Michaels commented that most pathologists should be able to recognise the characteristic histological features of this condition on fine needle aspiration cytology (FNAC). Mr Jeannon commented on the current trend towards open biopsy of parotid lumps in order to exclude lymphoma, in which case FNAC reveals a mixed lymphocytic picture. The role of computed tomography guided core-needle biopsy was also discussed. The general opinion of the meeting was that an adequate tissue sample should be acquired from parotid lumps to enable a pathological diagnosis to be made without the need for superficial parotidectomy.

\section{Synovial sarcoma of the neck presenting as a thyroid mass}

N C Modi, G Bruch, R Simo

From Guy's and St Thomas' Hospital, London, UK

\section{Case report}

A 39-year-old man presented with a rapidly enlarging neck lump at the upper pole of the left thyroid lobe. The rest of the otorhinolaryngological examination was normal. Thyroid function tests and calcitonin levels were normal and vanillylmandelic acid (VMA) testing was negative. A nuclear thyroid scan with uptake showed a large, cold nodule, and fine needle aspiration cytology of the lesion suggested either a spindle cell tumour or a medullary thyroid carcinoma. A computed tomography scan demonstrated a tumour arising from the upper pole of the left thyroid lobe, displacing the larynx and pharynx. There was no evidence of metastatic disease in the neck or lung. The patient underwent a total thyroidectomy and level six selective neck dissection. At the time of surgery, the tumour was found to be infiltrating the submucosal layer of the pharynx, which was resected. Histological analysis showed a biphasic synovial sarcoma with keratin-positive cells and diffuse bcl-2 oncogene product. The tumour was found to be separated from the thyroid by fibrous tissue, and markers for medullary carcinoma were negative.

\section{Discussion}

Synovial sarcoma is a rare soft tissue malignancy of mesenchymal origin, which usually occurs in the lower extremities of young adults. Only 3 per cent of cases occur in the head and neck. There have been no previous reported cases presenting as carcinoma of the thyroid. Diagnosis can be difficult and require immunohistochemistry, electron microscopy and chromosomal-translocation analysis. Treatment of synovial sarcoma usually involves surgery with adequate excision margins. The role of chemotherapy and radiotherapy is still debated. The prognosis is poor because of the occurrence of distant metastasis, especially to the lungs.

\section{A challenging neck mass}

S Mansuri, A Sandison, D J Howard

From the Charing Cross Hospital, London, UK

\section{Case report}

A 21-year-old Greek lady presented with a neck mass and increasing shortness of breath. On examination, she was euthyroid and had a $2 \times 2 \mathrm{~cm}$ neck mass on the right. She had been investigated in Greece, where a magnetic resonance imaging scan had been reported as showing a right thyroid mass with invasion of the adjacent trachea, causing 90 per cent obstruction. Fine needle aspiration cytology had demonstrated possible papillary cell carcinoma. She had been referred to the UK for specialist management. In the UK, the patient underwent a subtotal thyroidectomy and tracheal resection. Intra-operative review of the tracheal lumen demonstrated a pale lesion arising from the wall and invading out, not characteristic of a papillary carcinoma. The trachea was reconstructed using a homograft overlying a Silastic ${ }^{\circledR}$ stent. Histological analysis revealed a primary tracheal malignancy of the adenoid cystic carcinoma subtype. The patient made an uncomplicated recovery with a quick return to normal activity, and at the time of writing was awaiting radiotherapy.

\section{Discussion}

Primary tracheal tumours are rare, with an incidence of 0.2 per 100000 persons per year. Adenoid cystic carcinoma, the second commonest subtype, has an insidious growth but can metastase to lung and bone. Reconstruction following a large tracheal resection provides a challenging problem, for which a homograft offers an excellent solution. These preserved cadaveric specimens do not necessitate immunosupression, and are quickly lined with respiratory epithelium. Good results have been reported, with an adult UK series shortly to be published. 


\section{Rhinology session}

Chairman: Mr David Roberts

\section{Premaxillary mucous cyst and frontal mucocele after rhinoplasty}

G Kumar, K Young, D Roy

From St John's Hospital, Chelmsford, UK

\section{Case report}

Managing post-operative complications after rhinoplasty can be a challenge. Although rare, several complications of rhinoplasty have been described in the literature, e.g. premaxillary mucous cyst. We present two complications which occurred following rhinoplasty: a premaxillary mucous cyst and a frontal mucocele. The premaxillary mucous cyst was excised via a combined approach, i.e. subperiosteal intra-oral approach and endoscopic intranasal approach via inter-cartilaginous incision. The frontal mucocele was drained endoscopically via an intranasal approach. Frontal mucocele has not previously been reported as a complication of rhinoplasty.

\section{Discussion}

Mr Roberts agreed that these were rare cases. Premaxillary mucous cysts result from entrapment of mucosal remnants in the subcutaneous space. Meticulous extra-mucosal dissection and removal of mucosal debris from the operative site will prevent this complication. We prefer external osteotomy as this prevents mucosal entrapment. Premaxillary mucous cyst can be managed endoscopically via a buccal approach to avoid an external scar. The aetiology of frontal mucocele is ostial obstruction caused by an inflammatory, iatrogenic, traumatic or neoplastic process. The frontal mucocele could have been an incidental finding. However, the likely aetiology in our case was manipulation of the nasal bone after incomplete osteotomy, leading to inappropriate extension of the fracture line and frontal ostial blockage.

\section{Endoscopic modified Lothrop procedure for repair of lateral frontal sinus cerebrospinal fluid leak}

J K Anverali, A Hassaan, H A Saleh

From the Charing Cross Hospital, London, UK

\section{Case report}

Cerebrospinal fluid (CSF) leaks in the frontal sinus have always been a challenge to repair endoscopically. The superior and lateral aspects of the posterior table of the frontal sinus are the most difficult to reach, and have previously been repaired using open approach techniques, with their associated morbidities. We report a case of lateral frontal sinus CSF leak which was repaired using an endoscopic approach.

\section{Discussion}

The endoscopic modified Lothrop technique, normally used for intractable chronic frontal sinusitis which has failed other treatment, was used to approach this frontal sinus CSF leak in a poorly accessible area. To our knowledge, this is the first report of the use of this approach to repair a lateral frontal sinus CSF leak.

\section{Amelanotic melanoma of spenoethmoid sinuses}

A K Golagani, A Malik, M H Wickham

From the Barnsley District General Hospital, UK
Case report

A 64-year-old woman presented with intermittent epistaxis and a mass in the left nostril, associated with left-sided ptosis and diplopia. Computed tomography and magnetic resonance imaging scans showed a mass in the left sphenoid sinus extending into the left and right posterior ethmoidal cells and into the left nasal cavity. The mass was eroding the medial wall of the left orbit. Examination under anaesthesia was performed and a biopsy taken. Histopathological and immunohistochemical analysis confirmed the diagnosis of an amelanotic melanoma. This case was discussed in the multidisciplinary team meeting, and two doses of radiotherapy were given to the patient. Unfortunately, the patient died of a complicated gastric perforation during the course of radiotherapy.

\section{Discussion}

Primary melanocytic lesions arise from melanoblasts, which are resident in the mucosa of the nose and paranasal sinuses. Professor Michaels confirmed the diagnosis of amelanotic melanoma of the sphenoethmoid sinus. The treatment options for amelanotic melanoma at this site were discussed. Mr Roberts supported the treatment of radiotherapy in this case.

The meeting concluded that a multicentre, prospective study was required in order to assess objectively the optimal treatment regimen for mucosal melanoma, and in particular to define the role of the various adjuvant therapies available.

\section{Intracranial abscess from frontal sinusitis: a difficult diagnosis}

D Biswas, S Maini, D L Baldwin

From the Southmead Hospital, Bristol, UK

\section{Case report}

A 14-year-old boy presented with a one-week history of headache and vomiting. There was tenderness over the left frontal sinus. The white cell count was $15.9 / \mathrm{mm}^{3}$ and the C-reactive protein was $400 \mathrm{mg} / \mathrm{l}$. A computed tomography (CT) scan showed a fluid level in the left frontal sinus.

After four days, despite antibiotic treatment, a diffuse swelling developed on the left side of the forehead. At this point, magnetic resonance imaging showed a left frontal subperiosteal collection, which was subsequently aspirated under ultrasound guidance. After 12 days of treatment with intravenous antibiotics, there was complete resolution of physical signs and symptoms (white cell count $6.5 / \mathrm{mm}^{3}$, C-reactive protein $9 \mathrm{mg} / \mathrm{l}$ ) and the patient was discharged home.

The patient was reviewed five days after discharge. Although he remained symptom free, his mother was concerned about his unusual behaviour. A repeat CT scan showed an abscess in the left frontal lobe, which was drained on the same day (bi-coronal approach).

\section{Discussion}

Intracranial complications from frontal sinusitis may develop late, even after the infection has been eradicated clinically, and with normal biochemical and haematological parameters. We conclude that, in frontal sinusitis cases with unusual developments, a high index of suspicion, and repeat scanning without delay, is essential for early diagnosis of complications. 


\section{Recurrent frontal sinus abscess after osteoplastic surgery} for frontal sinus mucocele

I Friedrichs, A G Toma

From St George's Hospital, London, UK

\section{Case report}

A 46-year-old Malaysian man had been treated in 1978 in Malaysia for a frontal sinus mucocele, with a frontal osteotomy and enucleation via a bitemporal flap.

In 1988, he had presented with a painless swelling over his forehead, but declined any further surgery.

In August 2006, the patient represented with an abscess over his forehead. A computed tomography scan showed a left Pott's puffy tumour secondary to chronic rhinosinusitis. The patient's frontal sinuses involved the whole forehead, with multiple septi. The patient underwent an endoscopic left nasal polypectomy and sinus surgery. The frontal abscess was drained via a Lynch-Howard incision, and the fronto-nasal duct was left patent with a corrugated drain. Six weeks later, the patient presented with a recurrence of the Pott's puffy tumour. At revision surgery, the fronto-nasal duct was extensively widened. The Lynch-Howard incision allowed access to the very superior frontal sinus cells, establishing drainage of the abscess cavity.

\section{Discussion}

Professor Michaels confirmed the histological diagnosis of benign polyps, and Dr Connor confirmed the radiological diagnosis of a recurrent Pott's puffy tumour secondary to chronic rhinosinusitis. Based on this case report, $\mathrm{Mr}$ Roberts recommended an endoscopic drainage procedure, such as the modified Lothrop procedure, as the best treatment option.

\section{Paediatric session}

Chairman: Mr Sean Blaney

\section{Insidious proptosis in an eight-year-old child}

R S Natt, M Sivanandan, H Daya

From St George's Hospital, London, UK

\section{Case report}

An eight-year-old child presented with a four-week history of right eye swelling, with excessive epiphora and frontal headaches. Examination revealed significant proptosis and ophthalmoplegia. An urgent computed tomography scan demonstrated a right ethmoid sinus mass expanding the medial wall of the right orbit, both filling and obstructing drainage of the right maxillary sinus. A subsequent nasal biopsy was complicated by bleeding, requiring packing on three separate occasions. Histological analysis identified a psammomatoid ossifying fibroma. Magnetic resonance imaging confirmed the presence of a well defined, $4.5 \times$ $2.9 \mathrm{~cm}$ lesion arising from the right ethmoid region, with marked displacement of the globe laterally and deviation of the medial rectus. This extended superiorly to the cribiform plate, inferiorly to the maxillary crest and laterally to the medial antral wall. Magnetic resonance angiography identified the maxillary artery as the major blood supply, and embolisation was performed at Great Ormond Street Hospital. Surgical access to the tumour was achieved through a mid-facial degloving approach involving ENT, ophthalmology, maxillo-facial and neurosurgery teams. The patient made an uneventful recovery, with no evidence of recurrence at the time of writing.

\section{Discussion}

Psammomatoid ossifying fibromas, although benign, have a tendency towards locally aggressive behaviour. The requirement of embolisation prior to surgery emphasises the complexity of these tumours. A multi disciplinary team approach is imperative in achieving optimal surgical outcome and post-operative management of these patients.

An unusual cause of airway limitation initially diagnosed as asthma: myoepithelioma of the trachea

S Mahendran, N Gibbins, P Jani

From Addenbrooke's Hospital, Cambridge, UK

Case report

We present the first English literature report of biphasic stridor caused by a primary tracheal myoepithelioma. This is also the first report of this rare tumour occurring in the paediatric population.

\section{Discussion}

We discuss the treatment of this tumour and the novel approach of laser excision employed in this case. Although rare, primary tracheal myoepithelioma should be considered in the differential diagnosis of conditions presenting with irreversible airway obstruction, where a tracheobronchial mass is suspected.

Primary tracheal myoepithelioma can occur in the lung, but established literature ascribes the majority of these lesions to metastasis from primary cutaneous, breast and salivary myoepithelial tumours.

\section{Anomalous neck muscle in the anterior triangle presenting as a thyroglossal cyst \\ P Jani, N Gibbins, D Riches \\ From Addenbrooke's Hospital, Cambridge, UK}

Case report

We present the case of a supernumerary muscle in the anterior compartment of the neck, a condition known as rectus cervicalis, previously undescribed in the English electronic literature. A three-year-old boy presented with a history of recurrent infections of a mid-line neck lesion with an associated skin tag. This was excised under general anaesthetic. An unusual midline lesion was noted, consisting of a skin tag, a tubular tract and muscle bands running from a notched mandible to the manubrium.

\section{Discussion}

The anomalous muscle seen in this patient resembled the rectus abdominis muscle. Cases of rectus sternalis have been reported. We propose that this is the first recorded case of rectus cervicalis.

\section{Should we excise the thyroid gland?}

J Ahmed, L Cochrane, B Hartley

From the Great Ormond Street Hospital, London, UK

Case report

A 15-year-old girl presented to our institution with a diagnostic and management dilemma. She had a three-month history of a painless, left-sided, anterolateral cervical mass. Complete ENT examination was negative, apart 
from a firm, $2 \times 3 \mathrm{~cm}$ mass at neck level two. This had a mixed solid-cystic appearance on ultrasonography and magnetic resonance imaging. Fine needle aspiration cytology did however suggest probable paraganglioma or other neuroendocrine lesion. In view of this uncertainty, we performed an excision biopsy which, surprisingly, revealed papillary thyroid carcinoma within a thyroglossal duct remnant. The patient subsequently underwent total thyroidectomy, anterior neck clearance and radio-iodine ablation. The thyroid gland was histologically benign.

\section{Discussion}

Papillary thyroid carcinoma arising from a thyroglossal duct remnant is a rare entity, with about 250 cases reported worldwide. Most present in adulthood. Management is controversial. Options range from wide local excision to more radical surgery, including total thyroidectomy and radio-iodine ablation. Proponents of conservative surgery remark on the favourable prognosis with local clearance alone. In addition, to date there have been no reported paediatric cases of carcinoma in a concurrently excised thyroid gland. However, in our patient's particular case, it was felt unanimously that the course of received management was entirely appropriate. Her young age, the size of the lesion, its atypical location and the fact that clearance was not performed on initial operation made radio-iodine for ablation of residual disease a necessity. In conclusion, in such cases we advocate risk stratification and management similar to that for primary papillary thyroid carcinoma.

\section{Management of nasal dermoids with intracranial connection: a novel approach}

R L Heywood, L A Cochrane, B E J Hartley

From the Great Ormond Street Hospital for Children, London, UK

\section{Case report}

A male infant was noted to have a $0.5 \times 0.5 \mathrm{~cm}$ mass at the nasal tip at birth. An elective magnetic resonance imaging (MRI) scan was reported to be in keeping with a diagnosis of nasal dermoid with no intracranial extension. The patient subsequently presented with an abscess at the nasal bridge. An urgent computed tomography (CT) scan was reported as showing erosion of the glabellar region and anterior skull base. The abscess was incised and drained. A repeat CT scan showed a left-sided nasal dermoid extending into the extradural space of the anterior cranial fossa through a bony defect. Formal excision was performed via a combined approach with gull wing and external rhinoplasty incisions, with no post-operative complications.

\section{Discussion}

Controversies surround the optimal radiological investigation and surgical approach for cases of nasal dermoid. Radiological imaging is essential in order to delineate the anatomy and any intracranial extension. Magnetic resonance imaging and CT play complementary roles in imaging soft tissues and bony detail, respectively. Dr Connor was of the opinion that soft tissue analysis by MRI was the optimum first-line imaging modality to assess extent; bony defects seen on CT, suggestive of intracranial connection, are not specific for nasal dermoid. However, intracranial dermoid cannot be differentiated from fibrous tract by either modality. Formal craniotomy has traditionally been performed in the presence of intracranial extension. This can be avoided in cases of extradural dermoid by an approach from below with adequate access. 